УДК 621.434

\title{
3D Simulation of Gas Flow into the Formula Student Car Intake System
}

\author{
Barhm Mohamad ${ }^{a}$, \\ Jalics Karolya and Andrei A. Zelentsov*b \\ ${ }^{a}$ University of Miskolc \\ Miskolc, Hungary \\ ${ }^{b}$ Bauman Moscow State Technical University \\ Moscow, Russian Federation
}

Received 23.03.2020, received in revised form 28.04.2020, accepted 21.05.2020

Abstract. Formula Student Car (FS) is an international race car design competition for students at universities of applied sciences and technical universities. The winning team is not the one that produces the fastest racing car, but the group that achieves the highest overall score in design, racing performance. The arrangement of internal components for example, predicting aerodynamics of the air intake system is crucial to optimizing car performance as speed changes. The air intake system consists of an inlet nozzle, throttle, restrictor, air box and cylinder suction pipes (runners). The paper deals with the use of CFD numerical simulations during the design and optimization of components. In this research article, two main steps are illustrated to develop carefully the design of the air box and match it with the suction pipe lengths to optimize torque over the entire range of operating speeds. Also the current intake system was assessed acoustically and simulated by means of 1-D gas dynamics using the software AVL-Boost. In this manner, before a new prototype intake manifold is built, the designer can save a substantial amount of time and resources. The results illustrate the improvement of simulation quality using the new models compared to the previous AVL-Boost models.

Keywords: internal combustion engine, intake system, linear acoustic, geometry modification.

Citation: Mohamad B., Karoly Ja., Zelentsov A.A. 3D simulation of gas flow into the formula student car intake system, J. Sib. Fed. Univ. Eng. \& Technol., 2020, 13(5), 597-610. DOI: 10.17516/1999-494X-0249

(c) Siberian Federal University. All rights reserved

This work is licensed under a Creative Commons Attribution-Non Commercial 4.0 International License (CC BY-NC 4.0).

* Corresponding author E-mail address: pywand@gmail.com, zelentsov.aa@gmail.com 


\title{
Трехмерное моделирование течения газа
}

\section{во впускной системе автомобиля «Формулы Студент»}

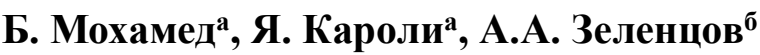 \\ ${ }^{a}$ Университет г. Мишкольияа \\ Венгрия, Мишкольи \\ ${ }^{\sigma}$ Московский государственный \\ технический университет им. Н.Э. Баумана \\ Российская Федераичи, Москва
}

Аннотация. «Формула Студент» - это международное соревнование по конструированию гоночных автомобилей для студентов университетов прикладных наук и технических университетов. Побеждает не та команда, которая создаст самый быстрый гоночный автомобиль, а группа, которая добивается наивысшего общего балла по конструированию, гоночным показателям. Например, расположение внутренних компонентов, прогнозирование аэродинамики системы впуска воздуха имеют решающее значение для оптимизации характеристик автомобиля на различных режимах работы. Система впуска воздуха состоит из впускного патрубка, дроссельной заслонки, ограничителя мощности (рестриктора), воздушной камеры и впускных каналов головки цилиндра. В статье рассматривается использование численного моделирования CFD при конструировании и оптимизации этих компонентов. Проиллюстрированы два основных шага, предпринимаемых для разработки конструкции воздушной камеры и сопоставления ее с длиной всасывающей трубы для оптимизации крутящего момента во всем диапазоне рабочих режимов. Также данная система впуска была оценена акустически и смоделирована с помощью 1-D газовой динамики с использованием программного обеспечения AVL Boost. Таким образом, перед созданием нового прототипа впускного коллектора проектировщик может сэкономить значительное количество времени и ресурсов. Результаты иллюстрируют улучшение качества протекания рабочего процесса двигателя при модификации его модели в AVL Boost.

Ключевые слова: двигатель внутреннего сгорания, впускная система, линейная акустика, изменение геометрии.

Цитирование: Мохамед, Б. Трехмерное моделирование течения газа во впускной системе автомобиля «Формулы Студент» / Б. Мохамед, Я. Кароли, А.А. Зеленцов // Журн. Сиб. федер. ун-та. Техника и технологии, 2020. 13(5). С. 597610. DOI: $10.17516 / 1999-494 X-0249$

\section{Введение}

Каждый год «Формула Студент» проводит всеобщее соревнование по проектированию для студентов инженерных специальностей со всего мира. На конкурсе оценивается конструкция, производительность и стоимость автомобиля заданного типоразмера, спроектированного в расчете на мелкосерийное производство. Цель данной исследовательской работы состоит в том, чтобы смоделировать и спроектировать впускную систему автомобиля «Формулы Студент» и, кроме того, продемонстрировать новые методики оптимизации такой системы в целях увеличения мощности автомобиля или снижения уровня шума.

Впускная система двигателя имеет три главных функции. Первая и наиболее определяющая функция - это обеспечение фильтрации воздуха от частиц пыли и примесей. Две другие характеристики, важные для инженеров, проектирующих впускную систему, - это расход 
воздуха и уровень шума. Расход воздуха через впускную систему прямо влияет на мощность, которую способен вырабатывать двигатель. Melaika в [1] показал влияние различных рестрикторов на производительность двигателя автомобиля «Формулы Студент», используя числовое моделирование в AVL BOOST. Были проведены исследования диаметров рестрикторов, которые изменялись от 15 до 60 мм. Меньший диаметр трубы впускного коллектора увеличивал гидравлическое сопротивление воздухозаборника и к тому же ухудшал наполнение цилиндра, результатом чего была более низкая мощность двигателя и более высокий удельный эффективный расход топлива. Mohamad [2] изучал влияние этанол-бензиновой топливной смеси на выходную мощность двигателя и выбросы вредных веществ. Результаты показали значительное улучшение процесса сгорания и характеристик выхлопных газов. Mohamad и Amroune [3] применили инструментарий вычислительной гидродинамики (CFD), чтобы описать влияние потока на уровень шума выхлопной камеры двигателя, и продемонстрировали потери в глушителе при разных частотах вращения коленчатого вала двигателя, используя 1D-моделирование в AVL Boost. Abdullah в [4] изучал эффективность работы двигателя с точки зрения расхода топлива. Давление воздуха на впуске влияло на выбросы вредных веществ с выхлопными газами. Результаты проведенных экспериментов показали, что на давление воздуха на впуске влияли степень открытия дроссельной заслонки и эффект Вентури, проявляющийся при подаче топлива в камеру сгорания карбюраторного двигателя. Без воздушного фильтра процесс сгорания протекает лучше вследствие более высокого давления воздуха на впуске, больше химической энергии топлива переходит в тепловую энергию, поэтому повышается температура выхлопных газов. Mohamad в [5] использовал метод матрицы переноса (transfer matrix method, TMМ), чтобы вычислить потери в глушителе, и этот алгоритм может также быть применен для других частей выхлопной системы. Результат изучения существующего глушителя сравнили с данными наблюдений, полученными во время экспериментов на автомобиле. Потери при передаче были оптимизированы для новой конструкции глушителя, при этом важную роль в проверке полученных результатов сыграла доступная литература. Acquati в [6] использовал уравнения баланса массы и импульса, чтобы смоделировать воздушный поток и давление для рабочих режимов; эти значения были рассчитаны с помощью моделей оперирующими осредненными значениями для впускной системы двигателя с искровым зажиганием. Как заметил Winterbone [7], в обычном двигателе внутреннего сгорания (ДВС) во время фазы впуска (впускной клапан открыт) объем цилиндра не заполняется полностью, как предполагается теоретически. Причина этого в изменении плотности воздуха и потерях давления в системе подачи. В результате этого процесса реальный коэффициент наполнения цилиндра и производительность двигателя в целом не совпадут с расчетными ожиданиями, если все эффекты не будут правильно учтены. Один из компонентов, который играет важную роль в процессе подачи воздуха, - это впускной коллектор (ВК). Его физические характеристики, такие как потери давления и неравномерность распределения этих потерь между впускными каналами в головке двигателя (несбалансированная подача воздуха), входят в число факторов, которые связаны с эффективностью потребления топлива и выбросами двигателя [8].

В нашем исследовании рассматривается улучшение параметров течения топливовоздушной смеси через впускной коллектор двигателя Honda CBR 600RR (PC 37) (рис. 1).

$$
-599-
$$




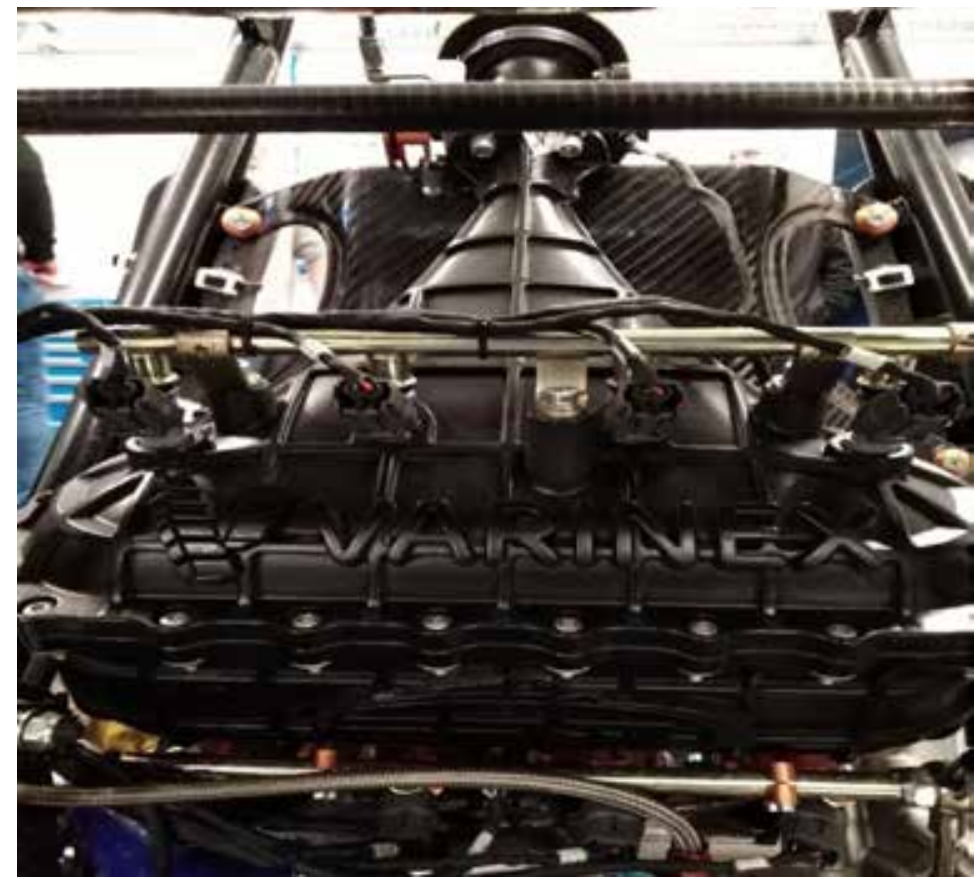

Рис. 1. Впускная система двигателя Honda CBR 600RR (PC 37)

Fig. 1. Honda CBR 600RR (PC 37) intake system

Довольно затруднительно получить входные параметры для CFD-анализа, необходимые для процесса проектирования и оптимизации, потому что невозможно использовать стационарный тип числового анализа. По этой причине в AVL-1D Boost Engine Simulation была создана одномерная модель двигателя, включающая системы впуска и выпуска.

Основные параметры воздушной камеры включают в себя длину впускных труб и каналов, а также общий объем воздушного коллектора. Впоследствии величины давления воздуха до коллектора генерируются с применением данного программного обеспечения. В дальнейшем эти значения используются как входные параметры для численного моделирования $\mathrm{CFD}$, которое проводят совместно с геометрической оптимизацией для обеспечения лучшей итоговой формы коллектора. Алгоритм процессов проектирования и оптимизации изображен на рис. 2.

\section{1. Численный анализ}

Для регулировки впускных патрубков и определения их оптимальной длины для двигателя «Формулы Студент» были применены уравнения волновой теории [9]. В результате получили следующее уравнение для длины патрубка:

$$
\text { Runner Length }=\left[\frac{\operatorname{ECD}(0.25)(2) \mathrm{V}_{\mathrm{w}}}{(\mathrm{RPM})\left(\mathrm{R}_{\mathrm{v}}\right)}-\frac{\mathrm{D}}{2}\right],
$$

где RPM - целевая скорость двигателя, об/мин; $\mathrm{R}_{\mathrm{v}}$ - номер отраженной волны $(1,2,3, \ldots \mathrm{n})$; D диаметр патрубка, мм; $\mathrm{V}_{\mathrm{w}}$ - рассчитанная скорость волны, мм/с. Эффективная длина камеры (ECD) определяется как [10] 


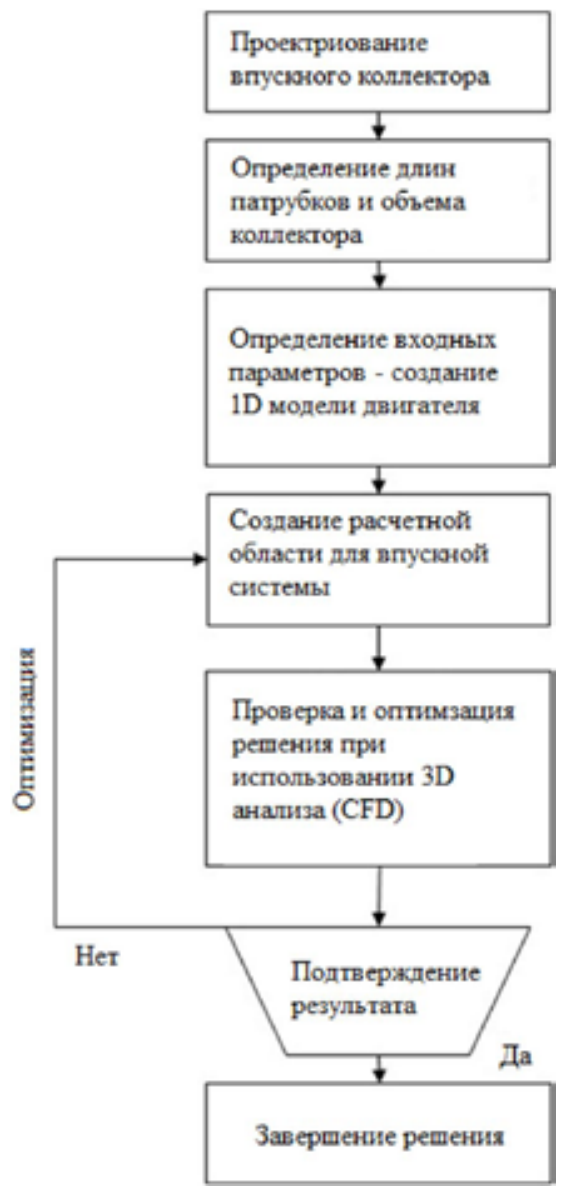

Рис. 2. Блок-схема процессов проектирования и оптимизации

Fig. 2. Flowchart of the design and optimization process

$$
\text { Effective Cam Duration }(E C D)=720^{\circ}-\text { Cam Duration }(\text { in degree })-30^{\circ} \text {. }
$$

Массовый расход через рестриктор $\left[\mathrm{m}_{\max }\right]$ вычисляют следующим образом:

$$
\mathrm{m}_{\text {max }}=\mathrm{A}_{\mathrm{r}} \mathrm{P}_{\mathrm{o}} \sqrt{\frac{\mathrm{K}}{\mathrm{RT}_{\mathrm{o}}}}\left(\frac{2}{\mathrm{k}+1}\right)^{\frac{(\mathrm{k}+1)}{2(\mathrm{k}-1)}},
$$

где $\mathrm{A}_{\mathrm{r}}$ - площадь рестриктора, мм²$; \mathrm{P}_{\mathrm{o}}$ - эталонное давление [Pa]; $\mathrm{R}$ - газовая постоянная, равная 287,04 [Дж/(кг К)]; $\mathrm{T}_{\mathrm{o}}$ - эталонная температура [К]; К - это удельная теплоемкость; $\mathrm{k}$ - показатель степени, равный 1,4 .

Объемный расход $\mathrm{Q}_{\max }$ рассчитывают следующим образом:

$$
\mathrm{Q}_{\max }=\frac{\mathrm{m}_{\max }}{\rho}
$$

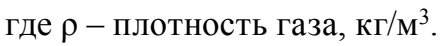

Аналитическое решение для уровня звукового давления (SPL) простой камеры, которая рассматривается как камера расширения, может быть получено либо методом потенциальной функции, либо методом матрицы переноса, как указано в уравнении [11]

$$
-601-
$$




$$
\operatorname{SPL}(\mathrm{dB})=20 \log _{10}\left(\frac{\mathrm{P}}{\mathrm{P}_{\mathrm{o}}}\right),
$$

где Р - это давление [Рa], $\mathrm{P}_{\text {o }}$ эталонное давление [Рa].

Математическая модель, используемая для трехмерного расчета потока на впуске и выпуске, основана на фундаментальных уравнениях трехмерного нестационарного переноса: уравнения количества движения (Навье-Стокса), энергии (Фурье-Кирхгофа) и сохранения массы (неразрывности), которые после процедуры осреднения по методу Фавра принимают форму Рейнольдса:

$$
\begin{aligned}
& \bar{\rho} \frac{\mathrm{D} \overline{\mathrm{W}}_{\mathrm{i}}}{\mathrm{Dt}}=\overline{\mathrm{G}}_{\mathrm{i}}-\frac{\partial \overline{\mathrm{P}}}{\partial \mathrm{x}_{\mathrm{i}}}+\frac{\partial}{\partial \mathrm{x}_{\mathrm{j}}}\left[\mu\left(\frac{\partial \overline{\mathrm{W}}_{\mathrm{i}}}{\partial \mathrm{x}_{\mathrm{j}}}+\frac{\partial \overline{\mathrm{W}}_{j}}{\partial \mathrm{x}_{\mathrm{i}}}-\frac{2}{3} \delta_{\mathrm{ij}} \frac{\partial \overline{\mathrm{W}}_{\mathrm{k}}}{\partial \mathrm{x}_{\mathrm{k}}}\right)-\bar{\rho} \overline{\mathrm{W}^{\prime}} \overline{\mathrm{W}}^{\prime}{ }_{\mathrm{j}}\right], \\
& \bar{\rho} \frac{\mathrm{D} \overline{\mathrm{H}}}{\mathrm{Dt}}=\overline{\mathrm{G}}_{\mathrm{j}} \overline{\mathrm{W}}_{\mathrm{j}}+\frac{\partial \overline{\mathrm{P}}}{\partial \mathrm{t}}+\frac{\partial}{\partial \mathrm{x}_{\mathrm{i}}}\left(\overline{\mathrm{\tau}}_{\mathrm{ij}} \overline{\mathrm{W}_{\mathrm{j}}}\right)+\frac{\partial}{\partial \mathrm{x}_{\mathrm{j}}}\left(\lambda \frac{\partial \overline{\mathrm{T}}}{\partial \mathrm{x}_{\mathrm{j}}}-\mathrm{c}_{\mathrm{p}} \bar{\rho}{\overline{\mathrm{T}}{ }^{\prime} \mathrm{W}^{\prime}}_{\mathrm{j}}\right), \\
& \frac{\partial \bar{\rho}}{\partial \mathrm{t}}+\frac{\partial}{\partial \mathrm{x}_{\mathrm{j}}}\left(\bar{\rho} \overline{\mathrm{W}}_{\mathrm{j}}\right)=0,
\end{aligned}
$$

где $\mathrm{W}_{\mathrm{i}}, \mathrm{W}_{\mathrm{j}}, \mathrm{W}_{\mathrm{k}}$ - проекции вектора скорости, м/с; $\mathrm{p}$ - давление, $\mathrm{H} / \mathrm{M}^{2} ; G_{i}$ - проекция вектора плотности объемных сил $\left(\mathrm{H} / \mathrm{M}^{3}\right)$ на ось $\mathrm{Ox}_{\mathrm{i}}$ прямоугольной декартовой системы координат; $H$ полная удельная энергия, Дж/кг; Т - температура, К; $\mu$ - динамическая вязкость, кг/(мс); $c_{p}-$ теплоемкость при постоянном давлении, Дж/(кг КК); $\lambda$ - теплопроводность, Вт/(м·К); $\delta_{i j}-$ символ Кронекера; $\mathrm{t}$ - время, с; $\frac{D}{D \tau}$ - субстанциональная производная. В уравнениях (6) использовано представление параметра $\Phi$ (может быть скоростью $\mathrm{W}$, давлением p, энтальпией Н и т.п.) как суммы осредненного $\bar{\Phi}$ и пульсационного $\Phi^{\prime}$ значений.

Система уравнений переноса в форме Рейнольдса (6) замыкается $k$ - - - - -моделью турбулентности, специально разработанной и верифицированной для процессов течения, сгорания и теплообмена в поршневых двигателях $[12,13]$. Она состоит из трех уравнений - для кинетической энергии турбулентности $k$, для скорости диссипации $\varepsilon$ этой энергии, известных из $\mathrm{k}-\varepsilon$ модели турбулентности, а также уравнения для нормированного масштаба скорости $\zeta=\bar{w}^{2} / k$. Модель турбулентности $k$ - $-f$, предложенная К. Ханиаличем (К. Hanjalič) и др. [14], содержит эллиптическую функцию f Дурбина (П.А. Дурбин), учитывающую пристеночную анизотропию турбулентности.

Систему уравнений (6) применяют для описания, соответственно, скорости потока (уравнение Навье-Стокса), энтальпии (уравнение энергии) и массы или плотности (уравнение неразрывности) в каждом контрольном объеме рассматриваемой расчетной области. Теплообмен в пристеночных областях определяют через толщину пограничного слоя с использованием гибридных пристеночных функций [15].

Также можно отметить, что данная математическая модель типична для CFD-расчетов процессов в поршневых двигателях и детально описана в трудах Merker и др. [16], Basshuysen и Schäfer [17], Кавтарадзе и др. [18]. 


\section{2. Разработка модели САE}

Трехмерная модель базовой впускной системы гоночных автомобилей «Формулы Студент» была построена на основе конкретного прототипа двигателя с использованием передового программного обеспечения для проектирования Creo 4.0, включая рестриктор, впускные каналы, соединенные с коллектором и впускным трубопроводом. Поперечное сечение и размеры впускного коллектора даны на рис. 3.
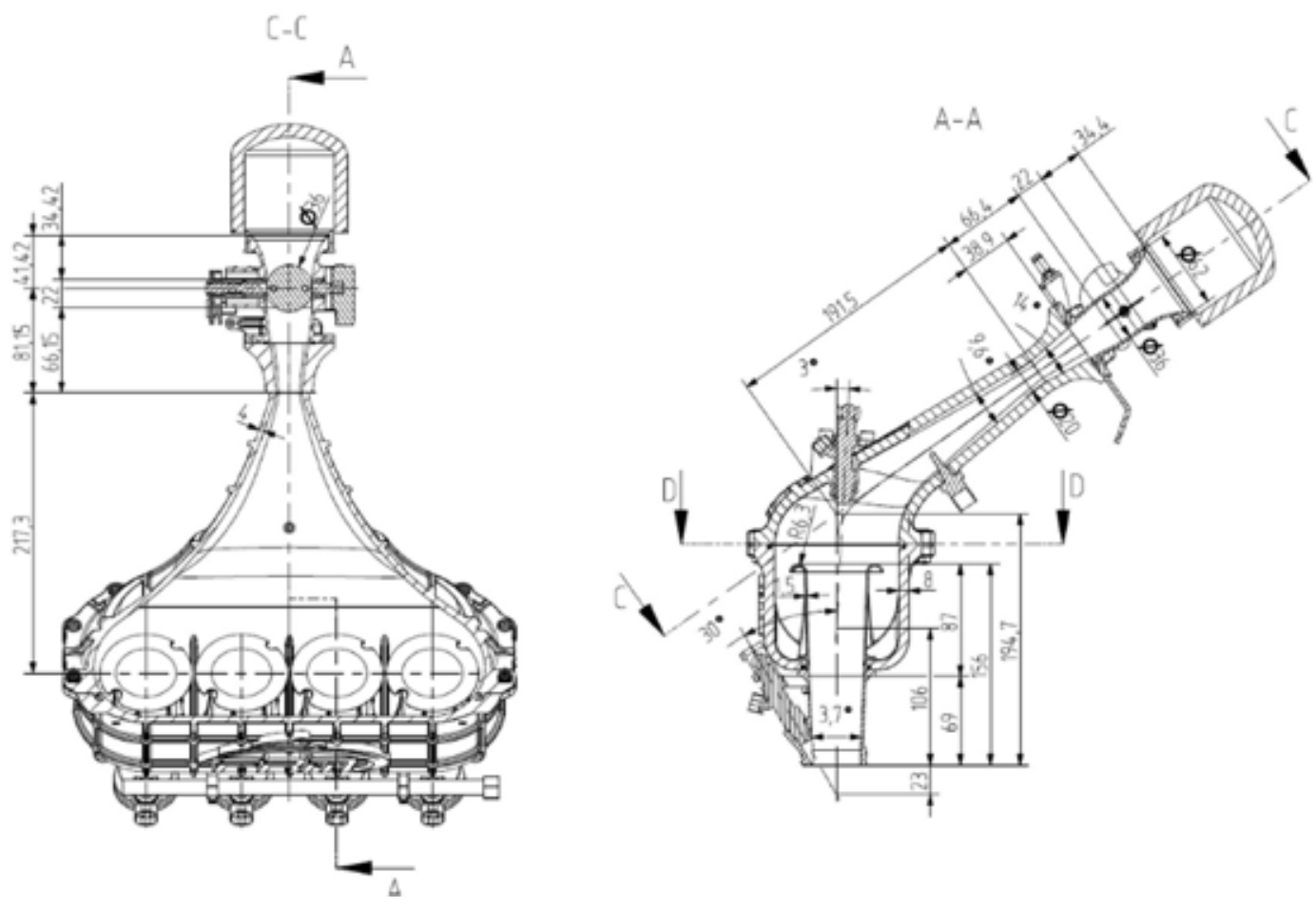

Рис. 3. Размеры впускной системы двигателя гоночного автомобиля «Формулы Студент»

Fig. 3. FS race car engine intake system dimensions

Это исследование было выполнено с использованием заводских данных четырехтактного четырехцилиндрового двигателя с искровым зажиганием Honda CBR 600RR (PC 37). Подробная спецификация базового двигателя, выбранного для моделирования, приведена в табл. 1.

Расчетный объем (внутренний объем впускного коллектора, трубопровода и патрубков до каждого цилиндра) был получен из твердотельной модели с помощью программного комплекса SolidWorks. Конструкция рестриктора и впускного трубопровода была исследована с использованием программного обеспечения для анализа и визуализации потока жидкости в процессе проектирования (CFD).

\section{3. Аналитические данные}

Оптимизация формы впускного коллектора выполняется с помощью CFD-моделирования (программное обеспечение 3D FIRE Advanced Flow). В целях моделирования и геометрической 
Таблица 1. Спецификация базового двигателя

Table 1. The specification of the base engine

\begin{tabular}{|c|c|c|c|c|c|}
\hline Трансмиссия & $\begin{array}{c}\text { Единицы } \\
\text { измерения }\end{array}$ & & & & \\
\hline Производитель / Модель & & \multicolumn{4}{|c|}{ Honda CBR600 RR (PC37) } \\
\hline Цилиндры \& Топливо & & Цилиндры: & 4 & Тип топлива: & АИ-98 \\
\hline $\begin{array}{l}\text { Рабочий объем \& Степень } \\
\text { сжатия }\end{array}$ & & $\begin{array}{c}\text { Рабочий } \\
\text { объем }\left(\mathrm{cm}^{3}\right) \text { : }\end{array}$ & 599 & $\begin{array}{c}\text { Степень } \\
\text { сжатия (_:1): }\end{array}$ & $12,2: 1$ \\
\hline $\begin{array}{l}\text { Диаметр поршня \& Ход } \\
\text { поршня }\end{array}$ & MM & $\begin{array}{l}\text { Диаметр } \\
\text { поршня: }\end{array}$ & 67 & Ход поршня: & 42,50 \\
\hline Длина шатуна & MM & \multicolumn{4}{|c|}{91} \\
\hline Привод клапанов & & \multicolumn{4}{|c|}{ Цепной, DOHC } \\
\hline Диаметры клапанов & MM & Впускной & 27,5 & Выпускной & 23 \\
\hline \multirow{4}{*}{ Фазы газораспределения } & \multirow{4}{*}{${ }^{\circ} \Pi К В$} & \multirow{2}{*}{ Впуск } & Открытие & $\begin{array}{c}\text { при высоте } \\
\text { подъема } 1 \text { мм }\end{array}$ & $22^{\circ}$ до ВМТ \\
\hline & & & Закрытие & $\begin{array}{c}\text { при высоте } \\
\text { подъема } 1 \text { мм }\end{array}$ & $43^{\circ}$ за НМТ \\
\hline & & \multirow{2}{*}{ Выпуск } & Открытие & $\begin{array}{c}\text { при высоте } \\
\text { подъема } 1 \text { мм }\end{array}$ & $40^{\circ}$ до НМТ \\
\hline & & & Закрытие & $\begin{array}{c}\text { при высоте } \\
\text { подъема } 1 \text { мм }\end{array}$ & $5^{\circ}$ за ВMT \\
\hline Порядок работы цилиндров & & \multicolumn{4}{|c|}{$1-2-4-3$} \\
\hline Давление топлива & бар & \multicolumn{4}{|c|}{4,00} \\
\hline
\end{tabular}

оптимизации расчетная область была разбита на 3115552 контрольных объема (КО). Некоторые основные параметры и характеристики модели приведены в табл. 2.

Все полученные из трехмерного сканирования геометрические параметры впускного коллектора и других частей двигателя были введены в 1D-модель (например, переменные диаметры впускного и выпускного трубопровода, различные по их длине, углы в сопряжениях отдельных участков впускного и выпускного трубопроводов, материалы с термическими свойствами и т.д.). Граничные условия задавались в соответствии с режимом работы двигателя, в качестве модели турбулентности использовалась k- - -f-модель.

Для получения граничных условий на впуске и выпуске, для дальнейшего расчета в трехмерной постановке, была создана одномерная модель двигателя (рис. 4).

Таблица 2. Характеристики модели

Table 2. Model properties

\begin{tabular}{|c|c|}
\hline Тип модели & $3 \mathrm{D}$ \\
\hline Тип сетки & Гексагональная \\
\hline Общее число элементов & 3115552 \\
\hline Объем модели & $0.004449 \mathrm{~m}^{3}$ \\
\hline Площадь поверхности & $0.346168 \mathrm{~m}^{2}$ \\
\hline
\end{tabular}




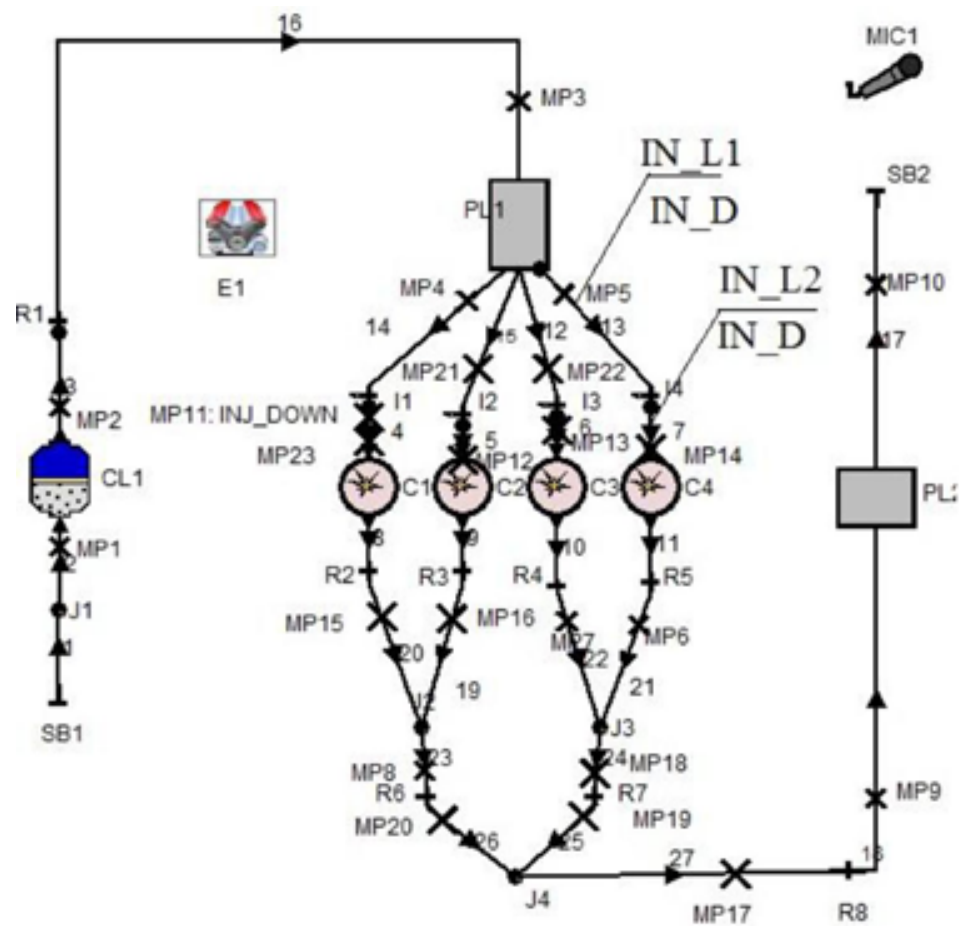

Рис. 4. Одномерная модель двигателя Honda CBR 600RR (PC 37)

Fig. 4. 1D model of the Honda CBR 600RR (PC 37) engine

Значения давления и температур для отдельных цилиндров двигателя в зависимости от времени были впоследствии получены из 1D-модели. Эти значения используются в качестве входных данных для анализа CFD. Окончательный анализ был выполнен как переходный процесс для полного цикла работы двигателя. Рассматривали несколько вариантов исполнения впускной системы двигателя для определения оптимальных размеров ее элементов (длины патрубков и объема впускного коллектора). При этом поиск оптимальной длины патрубков на впуске был проведен в диапазоне 150-300 мм, а для поиска оптимального общего объема коллектора был взят диапазон от 2 до 4 л.

\section{4. Результаты}

Расчеты и многократные итерации показали, что короткий впускной трубопровод отрицательно влияет на подачу воздуха, что объясняется повышением уровня турбулентности на ходе в цилиндры двигателя. Как следствие, происходит снижение мощности двигателя (рис. 5).

В версии, обозначенной как модификация 1, диаметр впускного канала IN_D был увеличен с 33,5 до 42 мм (см. схему двигателя, рис. 4), а объем впускного коллектора PL1 был уменьшен с 4 до 2 л. В версии, обозначенной как модификация 2, длина входного порта IN_L1 была увеличена со 100 до 250 мм, диаметр IN_D был уменьшен с 33,5 до 32 мм, а объем PL1 был уменьшен с 4 до 3 л.

Результаты показывают, что длинные впускные трубопроводы обеспечивают большой крутящий момент при низких оборотах двигателя, а короткие имеют преимущества при высо- 


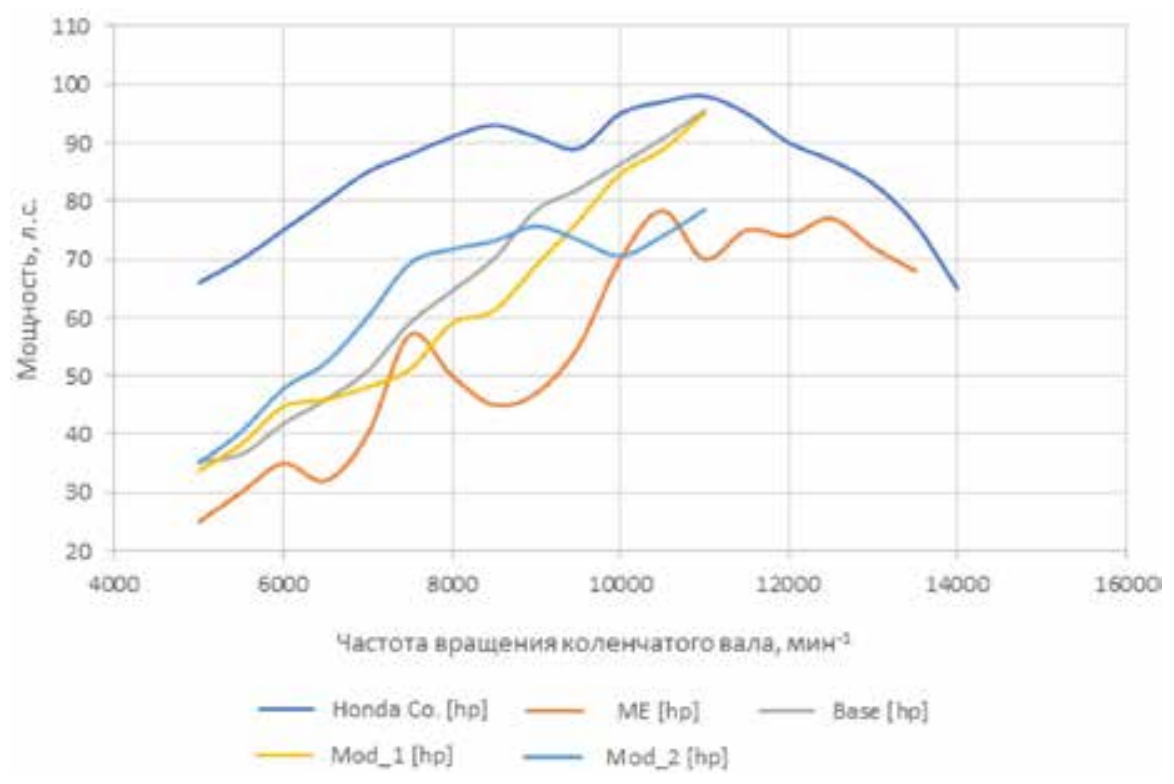

Рис. 5. Сравнение выходной мощности двигателя Нonda с базовым вариантом исполнения впускной системы (экспериментальные данные - ME, заводские - Honda Co.), с мощностью в базовом исполнении и модификациях 1 и $2(\operatorname{Mod} 1,2)$, полученными в результате расчетов

Fig. 5. Comparison power output from Honda Co., ME workshop test with the base case, and in the modifications 1 and 2 from software

ких оборотах двигателя. Что касается размера коллектора, небольшой объем камеры обеспечивает лучшую реакцию дроссельной заслонки, а большой объем позволяет достичь большей мощности двигателя (рис. 6).

Расчеты проводились в виде пошагового поиска вариантов с различными диаметрами, объемами и длинами. Критерием оптимальности была эффективная работа двигателя при разных режимах работы (5000-11000 об/мин). Подбор размеров впускной системы позволил снизить уровни звукового давления (SPL) от рабочего процесса двигателя (рис. 7).

Проведенный анализ осложняется необходимостью учета наличия высокоскоростного сжимаемого потока воздуха во впускной системе, перепадов давления и изменения температуры. Окончательное распределение скоростей, давлений и массовых потоков воздуха в воздушной камере приведено на рис. 8. Значения оцениваются на выходах из трубок камеры за один рабочий цикл двигателя (поворот коленчатого вала на $720^{\circ}$ ПКВ) при 8000 мин $^{-1}$. В качестве начальных условий задавали температуру и давление внутри рассматриваемого объема, а также кинетическую энергию турбулентности (данные были взяты из результатов расчета в Boost, рис. 9). 


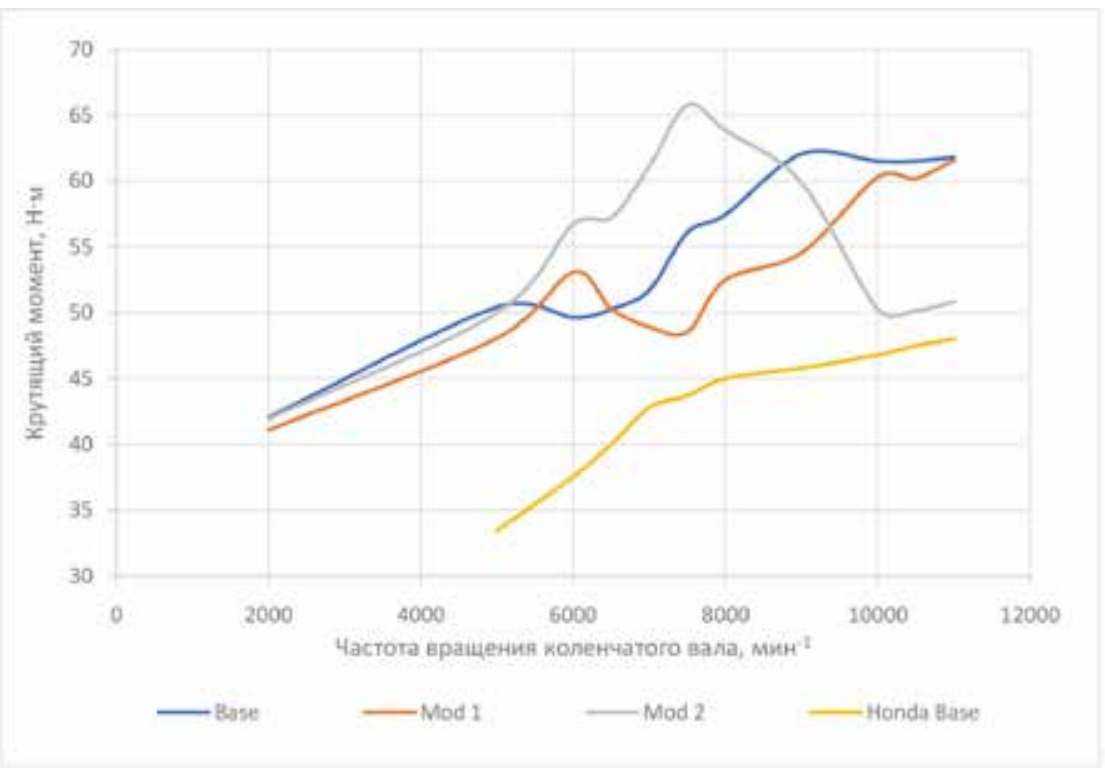

Рис. 6. Сравнение крутящего момента двигателя Honda с базовым вариантом исполнения впускной системы (экспериментальные данные - Honda Base, расчетные - Base), с модификациями 1 и 2 (Mod 1 , 2 - результаты расчетов)

Fig. 6. Comparison power output from Honda Co., ME workshop test with the base case, and in the modifications 1 and 2 from software

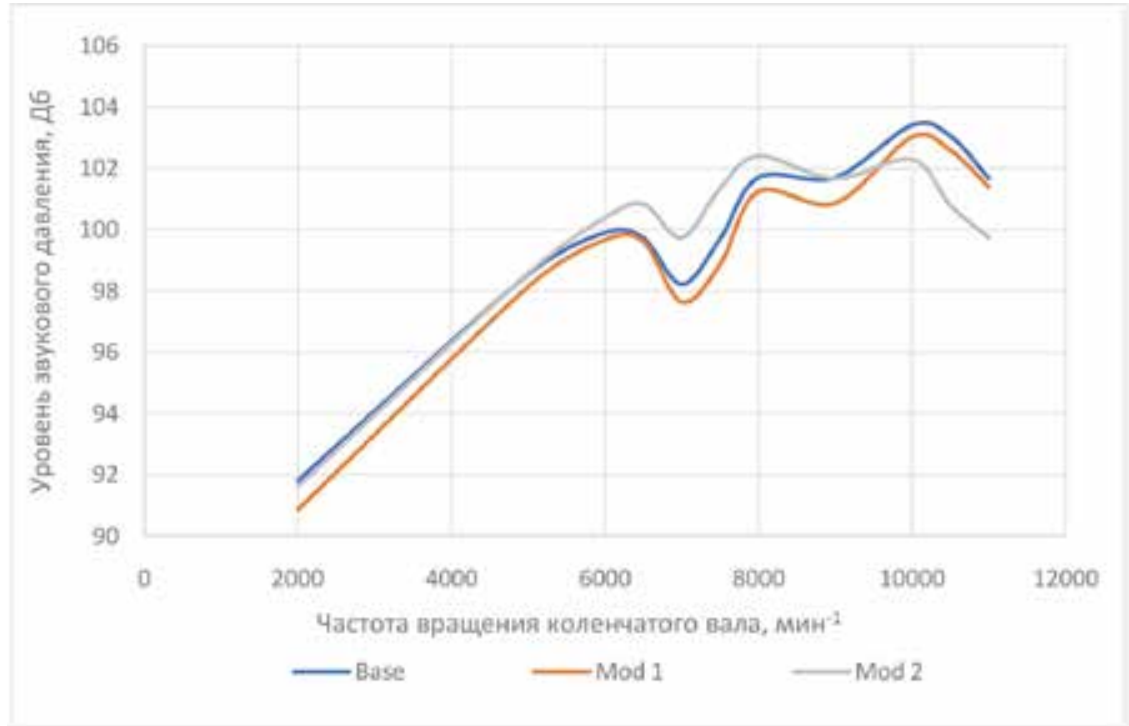

Рис. 7. Сравнение уровня звукового давления (SPL) на выпуске из двигателя Ноnda с базовым вариантом исполнения впускной системы (Base) с модификациями 1 и $2(\operatorname{Mod} 1,2$ - результаты расчетов)

Fig. 7. Comparison power output from Honda Co., ME workshop test with the base case, and in the modifications 1 and 2 from software 


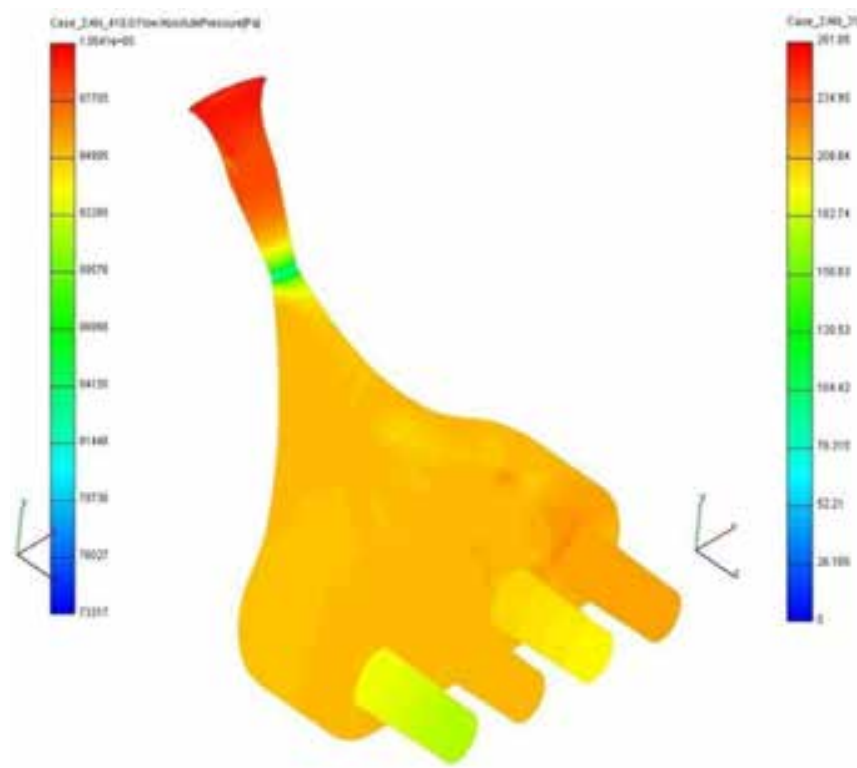

a

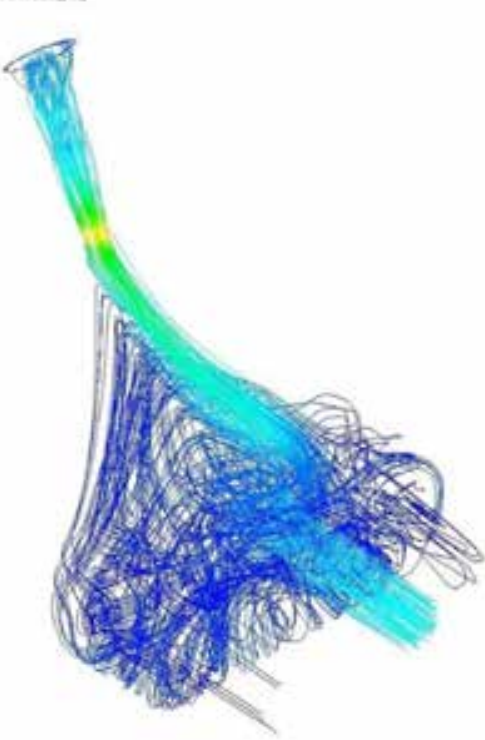

б

Рис. 8. Контур давления (a): Min=73317, Max=1.0041e+05 Ра при 8000 мин $^{-1}$; скорость потока (б): Min=0, $\mathrm{Max}=261.05 \mathrm{~m} / \mathrm{s}$ при 8000 мин $^{-1}$

Fig. 8. Left: Pressure contour, Min=73317, $\mathrm{Max}=1.0041 \mathrm{e}+05 \mathrm{~Pa}$ at $8000 \mathrm{rpm}$; Right: Velocity streamline Min=0, $\operatorname{Max}=261.05 \mathrm{~m} / \mathrm{s}$ at $8000 \mathrm{rpm}$

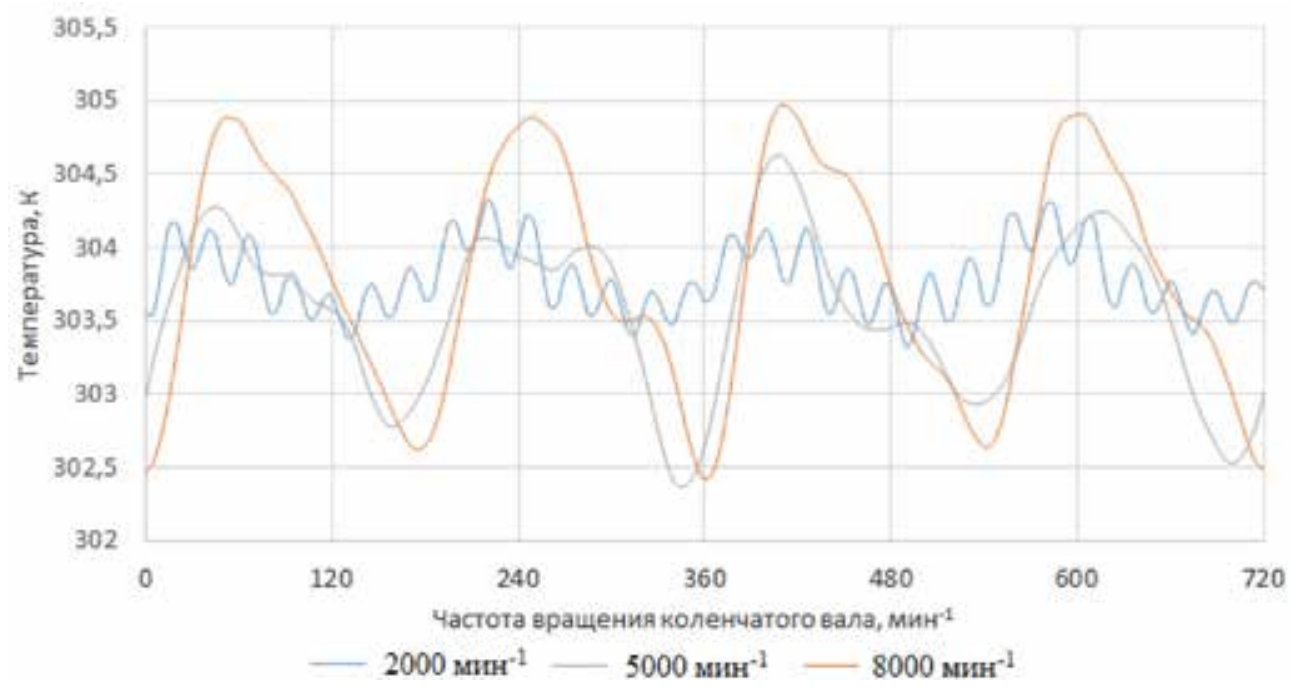

Рис. 9. Изменение температуры на рестрикторе при 2000, 5000 и 8000 мин $^{-1}$

Fig. 9. Temperature variation at restrictor of the intake system at 2000, 5000 and $8000 \mathrm{rpm}$

\section{Выводы}

Команда из Университета Мишкольц (Miskolc) усовершенствовала систему воздухозаборника автомобиля с использованием комбинированного одно- и трехмерного подходов, реализованных в AVL Boost и FIRE. Правила «Формулы Студент» ограничивают минимальный диа- 
метр рестриктора до 20 мм, что регулирует максимальный массовый расход воздуха на впуске. Камера ниже по потоку за рестриктором непосредственно влияет на количество свежего воздуха, попадающего в цилиндры. Слишком большая камера приводит к замедленной реакции двигателя на открытие дроссельной заслонки. Данное обстоятельство в сочетании с короткими всасывающими трубами является причиной получения на двигателе достаточного крутящего момента только при высоких скоростях вращения. Слишком маленькая камера ведет себя противоположно. Оптимальные длины впускных каналов, идентичных для каждого цилиндра, составили 250 мм при диаметре 32 мм, что соответствует варианту исполнения 2.

\section{Благодарности / Acknowledgements}

Авторы благодарят команду «Формула Студент» университета Мишкольц за помощь с проектированием, за методологию расчетов, а также доктора Ф. Хамада из Университета Тиссайд в Миддельсброте за комментарии, существенно улучшившие статью.

The authors thank Formula Racing Miskolc for assistance with design technique, methodology, and Dr. Faik Hamad, Teesside University in Middlesbrough, for comments that greatly improved the manuscript.

\section{Список литературы / References}

[1] Melaika, M., Rimkus, A., Vipartas, T., Air restrictor and turbocharger influence for the formula student engine performance, Procedia Engineering, 2017, 187, pp. 402-407.

[2] Mohamad, B., Szepesi, G., Bollo, B., Review Article: Effect of ethanol-gasoline fuel blends on the exhaust emissions and characteristics of SI engines, Vehicle and Automotive Engineering, 2018, 2, pp. 29-41.

[3] Mohamad, B., Amroune, S., The analysis and effects of flow acoustic in a commercial automotive exhaust system, Advances and Trends in Engineering Sciences and Technologies III, Proceedings of the 3rd International Conference on Engineering Sciences and Technologies (ESaT 2018), September 12-14, 2018, High Tatras Mountains, Tatranské Matliare, Slovak Republic, 2019, pp. 197-202.

[4] Adbulleh, N.R., Shahruddin, N.S., Mamat, A.M.I., Kasolang, S., Zulkifli, A., Mamat, R., Effects of air intake pressure to the fuel economy and exhaust emissions on a small SI engine, Procedia Engineering, 2013, 68, pp. 278-284.

[5] Mohamad, B., Karoly, J., Kermani, M., Exhaust system muffler volume optimization of light commercial passenger car using transfer matrix method, International Journal of Engineering and Management Sciences (IJEMS), 2019, 4, 132-139.

[6] Acquati, F., Battarola, L., Scattolini, R., Siviero, C., An intake manifold model for spark ignition engines, IFAC Proceedings, 1996, 29(1), pp. 7945-7950.

[7] Winterbone, E., Pearson, R., Horlock, J., Theory of engine manifold design: wave action methods for IC engines, Professional Engineering Publ. London, 2000.

[8] Byam, B., Fsadni, J., Hart, A., Lanczynski, R., An experimental approach to design, build, and test a throttle body and restrictor system for Formula SAE racing, SAE Technical Paper 2006-010748, SAE World Congress, Detroit, MI, USA. 2006.

[9] FSAE MQP, 2011, Retrieved from: http://moorsportsspares.com/file/2011_ttx25mkii_e.pdf (last access: 15.07.2019)

$$
-609-
$$


[10]Jawad, B., Lounsbery, A., Hoste, J. Evolution of intake design for a small engine formula vehicle, SAE Technical Paper, 2001-01-1211, SAE World Congress, Detroit, MI, USA, 2001.

[11] Shelagowski, M., Mahank, T. CFR Formula SAE intake restrictor design and performance, Proceedings of the 2015-ASEE North Central Section Conference American Society for Engineering Education, 2015.

[12]Tatschl, R., Schneider, J., Basara, D., Brohmer, A., Mehring, A., Hanjalic, K. Progress in the 3D-CFD calculation of the gas and water side heat transfer in engines, in 10. Tagung der Arbeitsprozess des Verbrennungsmotors (Proc. 10th Meeting on the Working Process of the Internal Combustion Engine), Graz, Austria, 2005.

[13]Tatschl, R., Basara, B., Schneider, J., Hanjalic, K., Popovac, M., Brohmer, A., Mehring, J. Advanced turbulent heat transfer modeling for IC-engine applications using AVL FIRE, Proceedings of International Multidimensional Engine Modeling User's Group Meeting, Detroit, USA, 2006.

[14]Hanjalic, K., Popovac, M., Hadziabdic, M. A robust near-wall elliptic-relaxation eddyviscosity turbulence model for CFD, International Journal of Heat and Fluid Flow, 2004, 25(6), pp. 1047-1051.

[15]Popovac, M., Hanjalic, K., Compound wall treatment for RANS computation of complex turbulent flow, Proc. 3rd M.I.T. Conference, Boston, USA, 2005.

[16]Merker, G., Schwarz, Ch., Teichmann R. Grundlagen Verbrennungsmotoren: Funktionsweise, Simulation, Messtechnik, 9th ed, Springer, Wiesbaden, 2019, P. 1117.

[17]Basshuysen, R., Schäfer, F. Handbuch Verbrennungsmotor, Vieweg und Sohn Verlag, Wiesbaden, 2007, p. 1032.

[18] Kavtaradze, R.Z., Onishchenko, D.O., Zelentsov, A.A., Sergeev, S.S., The influence of rotational charge motion intensity on nitric oxide formation in gas-engine cylinder, International Journal of Heat and Mass Transfer, 2009, 52(19-20), 4308-4316. 